\title{
Serum free fatty acids are associated with severe coronary artery calcification, especially in diabetes: a retrospective study
}

\author{
Yangxun Xin, Junfeng Zhang, Yuqi Fan ${ }^{*}$ and Changqian Wang ${ }^{*}$ (ID
}

\begin{abstract}
Background: Serum free fatty acid (FFA) concentrations are associated with coronary heart disease and diabetes mellitus (DM). Few studies focused on the relationship between serum FFA levels and coronary artery calcification (CAC).
\end{abstract}

Methods: This was a retrospective, single-centered study recruiting patients underwent FFA quantification, coronary angiography and intravascular ultrasound (IVUS). CAC severity was assessed with the maximum calcific angle (arc) of the calcified plaque scanned by IVUS. Patients with an arc $\geq 180^{\circ}$ were classified into the severe CAC (SCAC) group, and those with an $\operatorname{arc}<180^{\circ}$ were classified into the non-SCAC group. Clinical characteristics, serum indices were compared between 2 groups. Logistic regression, receiver operating characteristic (ROC) curves and area under the curves (AUC) were performed.

Results: Totally, 426 patients with coronary artery disease were consecutively included. Serum FFA levels were significantly higher in the SCAC group than non-SCAC group $(6.62 \pm 2.17 \mathrm{vs.} .13 \pm 1.73 \mathrm{mmol} / \mathrm{dl}, p<0.001)$. Logistic regression revealed that serum FFAs were independently associated with SCAC after adjusting for confounding factors in the whole cohort (OR 1.414, $\mathrm{Cl} 1.237-1.617, p<0.001)$, the non-DM group (OR 1.273, $\mathrm{Cl} 1.087-1.492, p=0.003)$ and the DM group (OR 1.939, Cl 1.388-2.710, $p<0.001)$. ROC analysis revealed a serum FFA AUC of 0.695 (Cl 0.641-0.750, $p<0.001$ ) in the whole population. The diagnostic predictability was augmented (AUC $=0.775, \mathrm{Cl} 0.690-0.859$, $p<0.001)$ in the DM group and decreased (AUC $=0.649, \mathrm{Cl} 0.580-0.718, p<0.001)$ in the non-DM group.

Conclusions: Serum FFA levels were independently associated with SCAC, and could have some predictive capacity for SCAC. The association was strongest in the DM group.

Keywords: Free fatty acids, Coronary arteries, Calcification, Intravascular ultrasound, Diabetes mellitus

\section{Background}

Coronary artery calcification (CAC) has been traditionally recognized as a common complication in aging patients, and those with diabetes mellitus (DM) or chronic kidney disease (CKD) $[1,2]$. CAC was observed in over $90 \%$ of men and $67 \%$ of women older than

\footnotetext{
*Correspondence: moricizine@163.com; wangcqcardiologist@126.com Department of Cardiology, Shanghai Ninth People's Hospital, Shanghai Jiao Tong University School of Medicine, Shanghai, China Co-corresponding author:Yuqi Fan
}

70 years $[3,4]$. The extent of CAC strongly correlates with the degree of atherosclerosis [5], and can predict future cardiovascular events [6-8]. Severe coronary artery calcification (SCAC), which indicates an extensively progressed calcified plaque typically with a calcified angle $>180^{\circ}$ surrounding the endothelium of the coronaries, remains a challenge for percutaneous coronary intervention (PCI). Clinical experience showed that SCAC poses a risk of failure in device delivery, coronary dissection, or insufficient expansion of the stent. Common 
methods to assess CAC include computed tomography $(\mathrm{CT})$, coronary angiography (CAG), intravascular ultrasound (IVUS) and optical coherence tomography (OCT) [9]. No effective pharmacotherapies have been confirmed to reverse the process of CAC.

Serum free fatty acids (FFAs) are one of the sources of energy in the body. They were found to be associated with insulin resistance and the development of DM [1013]. Previous work elucidated that serum FFAs were an independent risk factor for cardiovascular events both in stable coronary artery disease (CAD) $[14,15]$ and in acute coronary syndrome $[16,17]$. Serum FFA levels were also associated with prognosis in acute heart failure [18] and the incidence of heart failure in aged patients [19]. The underlying mechanism of the adverse cardiovascular prognosis of FFAs remains unknown. Moreover, whether FFAs exert a negative influence on coronaries by promoting the process of CAC needs to be investigated. Numerous studies have shown that FFAs (especially saturated FFAs) induce vascular calcification [20-25]. Few clinical studies focused on the relationship between serum FFA levels and arterial calcification [10, 22, 26], but with inconsistent conclusions.

Therefore, we attempted to explore the potential relationship between serum FFA levels and CAC in the present study.

\section{Methods}

\section{Study design and participants}

This was a single-center, retrospective study conducted at the Shanghai Ninth People's Hospital affiliated with Shanghai Jiao Tong University School of Medicine from February 2017 to February 2020. The enrollment criteria were as follows: (1) patients aged $\geq 18$ years, and (2) patients who underwent CAG and IVUS for a de novo lesion and were diagnosed with CAD. The exclusion criteria were as follows: (1) previous PCI or coronary artery bypass grafting (CABG) on the target vessel; (2) patients with a lesion of chronic total occlusion; (3) moderate or severe cardiac valve disease; (4) NYHA class 3-4 heart failure; (5) type $1 \mathrm{DM}$ (T1DM); (6) patients with a declined renal function of eGFR $\leq 30 \mathrm{ml} / \mathrm{min} / 1.73 \mathrm{~m}^{2}$ or who underwent hemodialysis; (7) severe hepatic dysfunction; (8) malignant tumor; or (9) patients with poor quality IVUS imaging or lack of laboratory test results. All patients gave written informed consent, and the study protocol was approved by the Institution's Human Investigation Committee. Procedures were performed in accordance with the Declaration of Helsinki.

\section{Demographic data and blood test}

Detailed medical history of the patients was collected after hospitalization. Weight and height were recorded and body mass index (BMI) was calculated by weight/ height ${ }^{2}\left(\mathrm{~kg} / \mathrm{m}^{2}\right)$. Blood pressure was measured on admission. Blood samples were routinely collected in the morning after overnight fasting when hospitalized, including lipid profiles (serum FFA was part of the fasting lipid profiles), kidney function, $\mathrm{HbA1c}$ et al. Estimated glomerular filtration rate (eGFR) were calculated according to CKDEPI equation.

\section{CAG and IVUS procedures}

The CAG procedures were performed according to generally accepted guidelines and routines [27, 28]. The radial artery was the preferred access approach. Lesions were imaged in at least two different projections, preferably at $90^{\circ}$. A lesion with a reduced luminal diameter of at least $50 \%$ was considered significant. All procedures were performed by two experienced interventional cardiologists, with sub-senior title or higher. Both the cardiologists decided together whether or not an IVUS procedure was needed after CAG. After intracoronary administration of $200 \mu \mathrm{g}$ of nitroglycerin, pre-intervention IVUS imaging of all the coronaries was performed using a commercially available IVUS system and catheter (iLab ${ }^{\mathrm{TM}}$ Ultrasound Imaging System, Boston Scientific Corp. Natic, MA, USA; Opticross ${ }^{\mathrm{TM}} 3.0 \mathrm{~F}$ intracoronary ultrasound catheter, Boston Scientific). The IVUS catheter was placed at least $10 \mathrm{~mm}$ distal to the lesion and then moved backward automatically at a speed of $0.5 \mathrm{~mm} / \mathrm{s}$ until it reached the coronary ostium.

\section{IVUS analysis}

All IVUS data were stored in DVDs and analyzed offline according to the criteria of the American College of Cardiology Clinical Expert Consensus Document on Standards for Acquisition, Measurement and Reporting of Intravascular Studies [29]. Analyses were independently performed with echo plaque software (Indec Medical Systems, Santa Clara, CA) by two experienced interventional cardiologists who were blinded to all the patients' characteristics. From all the coronaries, we selected the vessel with the most severe lesion and the most severe calcified plaque as our targets. In the case of multiple lesions within a single coronary segment, distinct lesions or stenosis had at least $5 \mathrm{~mm}$ distance between them, with the most severe lesion as our target. The lesion length was measured. The reference sites were the most normal-appearing positions within $5 \mathrm{~mm}$ proximal and distal to the lesion border but before any side branch, and were used to calculate a mean reference cross-sectional area (CSA). The minimum lumen CSA sites were the slices with the smallest lumen CSA. Quantitative analysis included the measurement of the external elastic membrane (EEM) and lumen area (LA) in the minimum 
Table 1 Baseline clinical characteristics

\begin{tabular}{|c|c|c|c|c|}
\hline Variable & Total $(n=426)$ & Non-SCAC $(n=320)$ & $\operatorname{SCAC}(n=106)$ & $P$ value \\
\hline Age, years & $67.14 \pm 10.29$ & $65.62 \pm 10.11$ & $71.74 \pm 9.44$ & $<0.001$ \\
\hline Male sex, \% & $281(66.0)$ & $213(66.6)$ & $68(64.2)$ & 0.650 \\
\hline $\mathrm{BMI}, \mathrm{kg} / \mathrm{m}^{2}$ & $24.40 \pm 2.84$ & $24.44 \pm 2.80$ & $24.29 \pm 2.98$ & 0.653 \\
\hline Smoking, \% & $221(51.9)$ & $170(53.1)$ & $51(48.1)$ & 0.371 \\
\hline Family history, \% & $103(24.2)$ & $75(23.4)$ & $28(26.4)$ & 0.535 \\
\hline Hypertension, \% & $303(71.1)$ & $221(69.1)$ & $82(77.4)$ & 0.102 \\
\hline Diabetes, $\%$ & $124(29.1)$ & $84(26.2)$ & $40(37.7)$ & 0.024 \\
\hline Prior Ml, \% & $42(9.9)$ & $30(9.4)$ & $12(11.3)$ & 0.560 \\
\hline Prior PCI \% & $90(21.1)$ & $62(19.4)$ & $28(26.4)$ & 0.124 \\
\hline Clinical presentation & & & & 0.980 \\
\hline SCAD & $354(83.1)$ & $266(83.1)$ & $88(83.0)$ & \\
\hline ACS & $72(16.9)$ & $54(16.9)$ & $18(17.0)$ & \\
\hline $\mathrm{eGFR}, \mathrm{ml} / \mathrm{min} / 1.73 \mathrm{~m}^{2}$ & $80.89 \pm 20.79$ & $82.51 \pm 20.64$ & $76.00 \pm 20.56$ & 0.005 \\
\hline $\mathrm{HbA} 1 \mathrm{c}, \%$ & $6.34 \pm 1.38$ & $6.26 \pm 1.33$ & $6.59 \pm 1.48$ & 0.031 \\
\hline $\mathrm{TG}, \mathrm{mmol} / \mathrm{L}$ & $1.63 \pm 0.89$ & $1.66 \pm 0.87$ & $1.54 \pm 0.94$ & 0.204 \\
\hline $\mathrm{TC}, \mathrm{mmol} / \mathrm{L}$ & $4.17 \pm 1.01$ & $4.14 \pm 0.97$ & $4.24 \pm 1.14$ & 0.381 \\
\hline $\mathrm{HDL}, \mathrm{mmol} / \mathrm{L}$ & $1.06 \pm 0.26$ & $1.05 \pm 0.26$ & $1.07 \pm 0.28$ & 0.465 \\
\hline $\mathrm{LDL}, \mathrm{mmol} / \mathrm{L}$ & $2.66 \pm 0.85$ & $2.63 \pm 0.82$ & $2.74 \pm 0.94$ & 0.230 \\
\hline Serum FFAs, mmol/dl & $5.51 \pm 1.96$ & $5.13 \pm 1.73$ & $6.62 \pm 2.17$ & $<0.001$ \\
\hline $\mathrm{SBP}, \mathrm{mmHg}$ & $131.50 \pm 17.02$ & $130.03 \pm 16.03$ & $135.93 \pm 19.10$ & 0.002 \\
\hline $\mathrm{DBP}, \mathrm{mmHg}$ & $75.01 \pm 10.52$ & $75.48 \pm 10.38$ & $73.62 \pm 10.89$ & 0.116 \\
\hline $\mathrm{PP}, \mathrm{mmHg}$ & $56.51 \pm 13.63$ & $54.55 \pm 12.55$ & $62.41 \pm 15.07$ & $<0.001$ \\
\hline \multicolumn{5}{|l|}{ Prior medical treatment } \\
\hline Anti-platelet drugs (n/\%) & $119(28.0)$ & $84(26.3)$ & $35(33.0)$ & 0.184 \\
\hline Statins (n/\%) & $99(23.2)$ & $73(22.8)$ & $26(24.5)$ & 0.717 \\
\hline ACEI/ARB (n/\%) & $194(45.5)$ & $139(43.4)$ & $55(51.9)$ & 0.130 \\
\hline$\beta$-blockers (n/\%) & $81(19.0)$ & $55(17.2)$ & $26(24.5)$ & 0.095 \\
\hline CCB $(n / \%)$ & $144(33.8)$ & $102(31.9)$ & $42(39.6)$ & 0.144 \\
\hline Insulin (n/\%) & $32(7.5)$ & $21(6.6)$ & $11(10.4)$ & 0.197 \\
\hline
\end{tabular}

Data are expressed as the mean \pm SD. BMI, body mass index; MI, myocardial infarction; $\mathrm{PCl}$, percutaneous coronary intervention; SCAD, stable coronary artery disease; ACS, acute coronary syndrome; eGFR, estimated glomerular filtration rate; TC, total cholesterol; TG, triglyceride; HDL, high-density lipoprotein cholesterol; LDL, lowdensity lipoprotein cholesterol; SBP, systolic blood pressure; DBP, diastolic blood pressure; PP, pulse pressure; ACEI/ARB, angiotensin-converting enzyme inhibitors/ angiotensin receptor blockers; $\mathrm{CCB}$, calcium channel blockers

lumen area (MLA) position. Plaque plus media CSA was calculated as MLA-EEM minus MLA-LA. Plaque burden was calculated as plaque and media CSA divided by MLA-EEM multiplied by $100 \%$.

Coronary calcium was defined as a brighter plaque than adventitia with acoustic shadowing. The arc of the calcified plaque in the lesion was measured. Calcium length was determined as the length of the calcified plaque with an arc. According to the arc value, the cohort was divided into two groups: (1) Patients with an arc $\geq 180^{\circ}$ were classified into the SCAC group, and those with a calcified $\operatorname{arc}<180^{\circ}$ or no calcium were classified into the nonSCAC group.

\section{Statistical analysis}

Statistical analysis was performed with SPSS version 20.0 (IBM Corp., Armonk, NY, USA). Continuous variables are expressed as the Mean \pm SD for normally distributed variables. Categorical data are presented as frequencies and proportions. Continuous variables were assessed using unpaired Student's t-tests, while categorical variables were compared using chi-square tests. Pearson's correlation analyses between serum FFAs, and clinical and IVUS data were performed. Binary logistic regressions were used to calculate the correlations between the confounders and SCAC. Parameters were considered potential confounders if associations were found with a $p$-value $<0.10$ in single factor analysis. Receiver operating characteristic (ROC) curves were constructed to assess the diagnostic value of FFAs according to the area under the curve (AUC). All $p$-values and confidence intervals (CI) were two-sided, and $p<0.05$ was considered statistically significant. 
Table 2 Angiographic and IVUS analysis of lesion characteristics

\begin{tabular}{|c|c|c|c|c|}
\hline Variable & Total $(n=426)$ & Non-SCAC $(n=320)$ & $\operatorname{SCAC}(n=106)$ & $P$ value \\
\hline Target vessel & & & & 0.066 \\
\hline LM & $14(3.3)$ & $9(2.8)$ & $5(4.7)$ & \\
\hline LAD & $311(73.0)$ & $226(70.6)$ & $85(80.2)$ & \\
\hline LCX & $31(7.3)$ & $28(8.8)$ & $3(2.8)$ & \\
\hline $\mathrm{RCA}$ & $70(16.4)$ & $57(17.8)$ & $13(12.3)$ & \\
\hline Lesion length (mm) & $22.59 \pm 12.63$ & $18.91 \pm 10.0$ & $33.73 \pm 13.25$ & $<0.001$ \\
\hline Reference EEM (mm²) & $14.39 \pm 4.46$ & $14.33 \pm 4.58$ & $14.59 \pm 4.06$ & 0.597 \\
\hline $\mathrm{MLA}-\mathrm{CSA}\left(\mathrm{mm}^{2}\right)$ & $14.46 \pm 4.55$ & $14.45 \pm 4.69$ & $14.50 \pm 4.12$ & 0.919 \\
\hline $\mathrm{MLA}-\mathrm{LA}\left(\mathrm{mm}^{2}\right)$ & $4.65 \pm 2.60$ & $4.92 \pm 2.79$ & $3.82 \pm 1.68$ & $<0.001$ \\
\hline Plaque area $\left(\mathrm{mm}^{2}\right)$ & $9.83 \pm 3.52$ & $9.57 \pm 3.53$ & $10.59 \pm 3.40$ & 0.010 \\
\hline Plaque burden & $0.69 \pm 0.11$ & $0.67 \pm 0.11$ & $0.73 \pm 0.08$ & $<0.001$ \\
\hline Calcium length (mm) & $7.18 \pm 5.32$ & $4.97 \pm 3.44$ & $11.36 \pm 5.73$ & $<0.001$ \\
\hline Calcium arc $\left(^{\circ}\right)$ & $159.49 \pm 107.78$ & $90.03 \pm 41.42$ & $290.56 \pm 62.91$ & $<0.001$ \\
\hline
\end{tabular}

Data are expressed as the mean \pm SD. LM, left main coronary; LAD, left anterior descending branch; LCX, left circumflex branch; RCA, right coronary artery; EEM, external elastic membrane; MLA-CSA, cross-sectional area in the minimal lumen area site; MLA-LA, luminal area in the minimal lumen area site

\section{Results}

\section{Baseline clinical characteristics}

Totally, 495 patients who underwent both CAG and IVUS were analyzed. A total of 69 patients were excluded, including 36 cases for previous PCI on target vessel, 2 for CABG of the target, 6 for severe heart failure, 1 for T1DM, 5 for declined renal function, 5 for cancer, 9 for insufficient lab results and 5 for poor quality IVUS. Finally, 426 vessels from 426 patients (SCAC group: 106, 24.9\%) were included. Table 1 shows that patients in the SCAC group were older $(71.74 \pm 9.44$ vs. $65.62 \pm 10.11$ years, $p<0.001)$, more likely to have a history of DM (37.7\% vs. 26.2\%, $p=0.024)$, elevated HbA1c $(6.59 \pm 1.48$ vs. $6.26 \pm 1.33, p=0.031)$, increased pulse pressure $(62.41 \pm 15.07$ vs. $54.55 \pm 12.55 \mathrm{mmHg}$, $p<0.001)$ and decreased renal function $(76.00 \pm 20.56$ vs. $\left.82.51 \pm 20.64 \mathrm{ml} / \mathrm{min} / 1.73 \mathrm{~m}^{2}, p=0.005\right)$. Serum FFA levels were significantly increased in the SCAC group $(6.62 \pm 2.17$ vs. $5.13 \pm 1.73 \mathrm{mmol} / \mathrm{dl}, p<0.001)$. No significant differences in clinical presentation, serum cholesterol levels, total triglyceride levels, or use of drugs were observed between the two groups (Table 1).

\section{CAG and IVUS results}

The distribution of SCAC in the coronaries was similar to that in the non-SCAC group (Table 2). There were no significant differences in MLA-EEM and reference EEM between the two groups. Patients with SCAC tended to have a severe lesion with an increased lesion length $(33.73 \pm 13.25$ vs. $18.91 \pm 10.0 \mathrm{~mm}, p<0.001)$, a smaller MLA-LA (3.82 \pm 1.68 vs. $\left.4.92 \pm 2.79 \mathrm{~mm}^{2}, p<0.001\right)$ and a higher plaque burden $(0.73 \pm 0.08$ vs. $0.67 \pm 0.11$, $p<0.001)$ than the non-SCAC patients. Calcium length
$(11.36 \pm 5.73$ vs. $4.97 \pm 3.44 \mathrm{~mm}, p<0.001)$ and calcium $\operatorname{arc}\left(290.56 \pm 62.91\right.$ vs. $\left.90.03 \pm 41.42^{\circ}, p<0.001\right)$ were also increased in the SCAC group (Table 2).

Correlation between serum FFAs and clinical and IVUS data Pearson's correlation analysis revealed that serum FFA levels were significantly correlated with plaque burden $(\mathrm{r}=0.157, p=0.001)$, lesion length $(\mathrm{r}=0.224, p<0.0001)$, calcium length $(\mathrm{r}=0.173, \quad p=0.002)$, calcium arc $(\mathrm{r}=0.353, p<0.001)$ and HbA1c $(\mathrm{r}=0.167, p<0.001)$. No positive relationship was found between serum FFAs and BMI ( $\mathrm{r}=0.032, p=0.505)$ (Fig. 1).

\section{Correlation between serum lipid profiles and the extent of coronary calcification}

No positive correlations were found between subfractions of serum lipid profiles (triglyceride, total cholesterol, high density lipoprotein, low density lipoprotein) and calcium parameters (calcium arc, calcium length of arc), except for a weak correlation between high density lipoprotein and calcium length $(\mathrm{r}=0.130, p=0.023)$, (Fig. 2).

\section{Risk factors of SCAC in the whole cohort and subgroups}

All the variables were compared with independent t-tests or chi-square test. Potential confounders with a $p$-value $<0.10$ combined with traditionally recognized risk factors of cardiovascular disease were entered into the logistic regression model. We built three models as presented in Table 3 and Fig. 3. Model 1 showed that in the whole cohort, after adjusting for pulse pressure, age, eGFR and HbA1c, serum FFA levels were independently associated with SCAC $(\mathrm{OR}=1.414,95 \%$ 

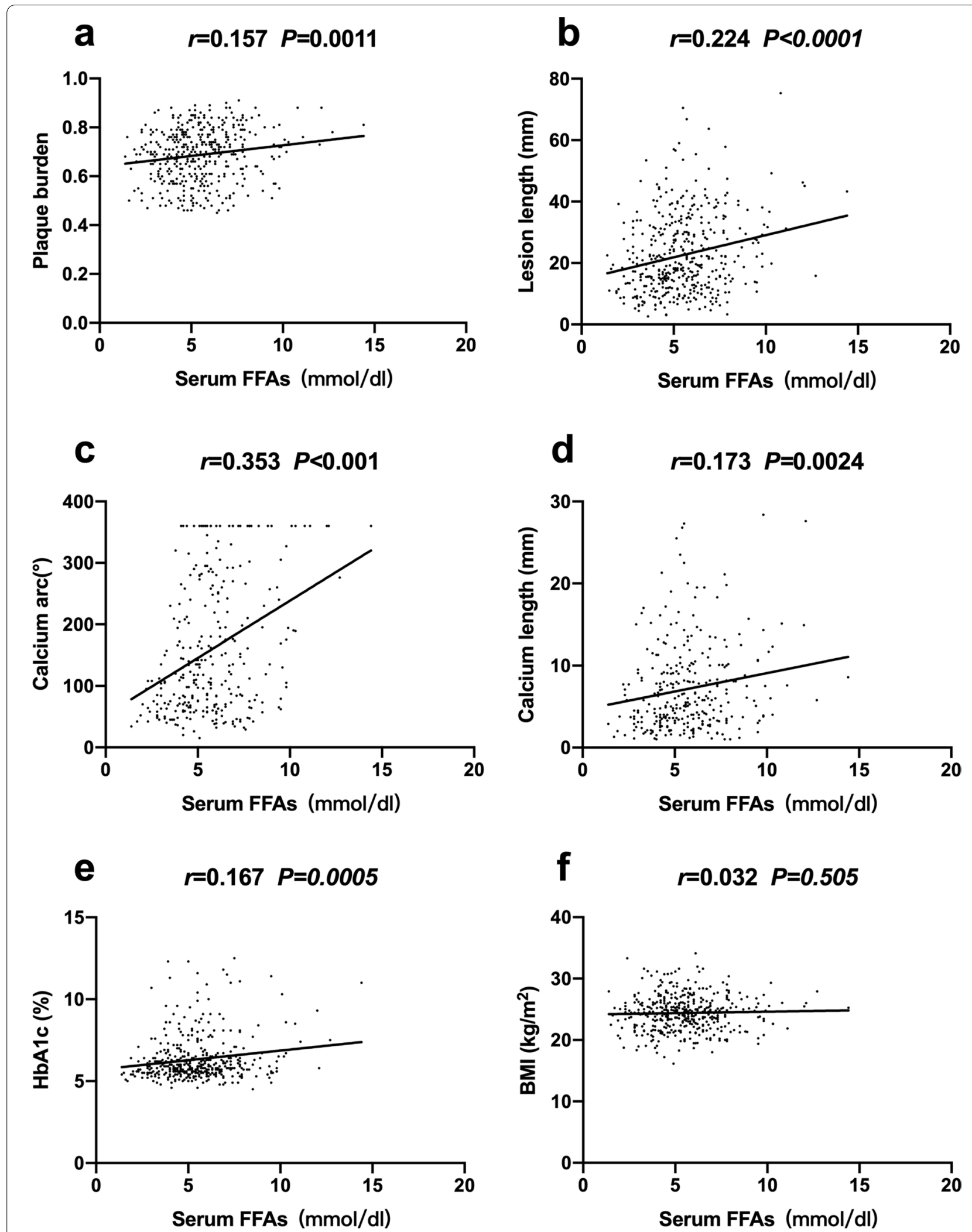

Fig. 1 Pearson correlation analysis of serum FFAs with plaque characteristics $(\mathbf{a}, \mathbf{b})$, coronary calcification severity (c, d), HbA1c (e) and BMI (f) 

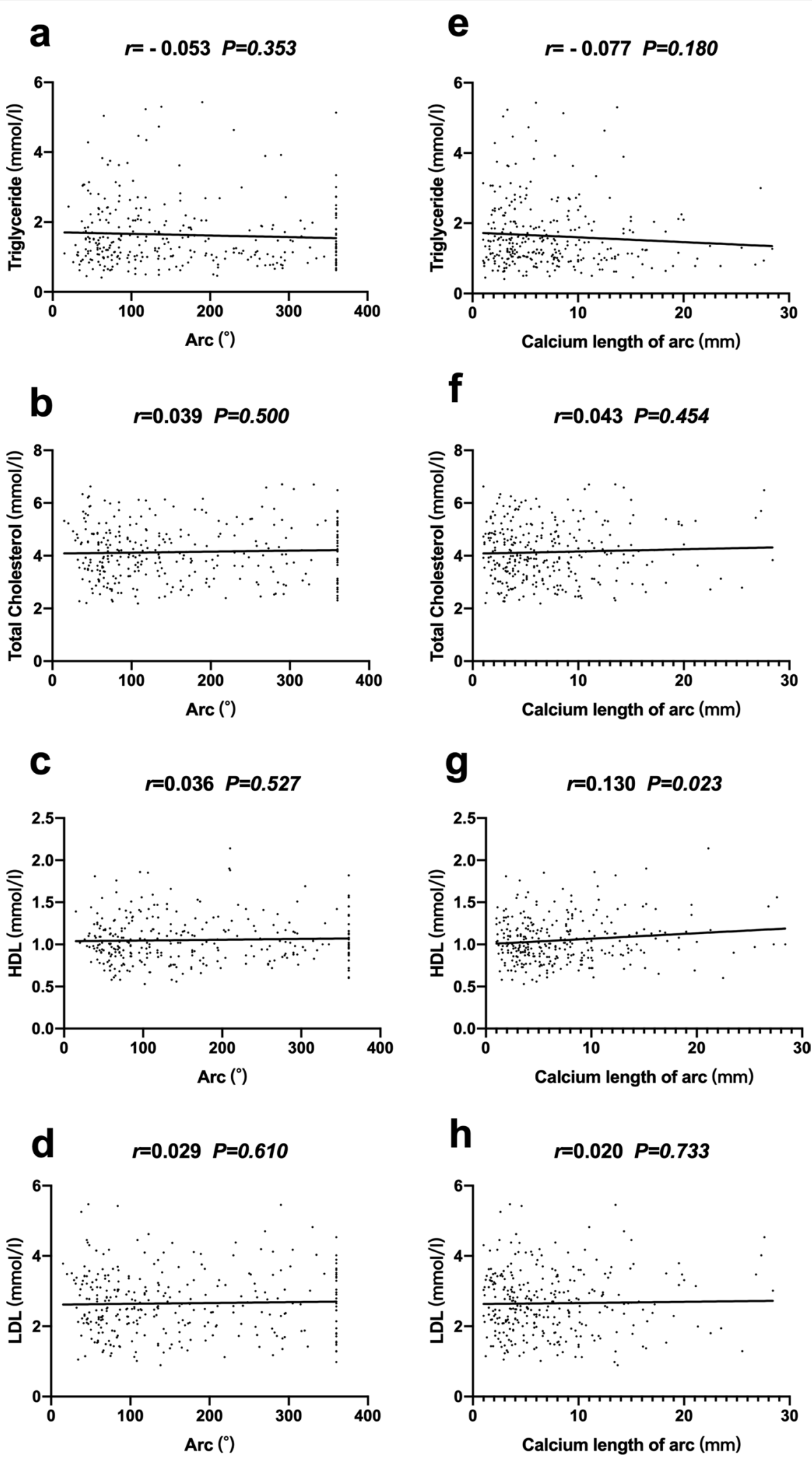

Fig. 2 Pearson correlation analysis between calcified parameters and subfractions of serum lipid profiles. Calcified arc with lipids (a, b, c, d), calcium length of arc with lipids $(\mathbf{e}, \mathbf{f}, \mathbf{g}, \mathbf{h})$ 
Table 3 Multivariate logistic regression for SCAC in the whole cohort (Model 1), non-DM group (Model 2), DM group (Model 3)

\begin{tabular}{|c|c|c|c|c|c|c|c|c|c|}
\hline & \multicolumn{3}{|c|}{ Model 1 (whole cohort) } & \multicolumn{3}{|c|}{ Model 2 (non-DM) } & \multicolumn{3}{|c|}{ Model 3 (DM) } \\
\hline & OR & $95 \% \mathrm{Cl}$ & Sig & OR & $95 \% \mathrm{Cl}$ & Sig & OR & $95 \% \mathrm{Cl}$ & Sig \\
\hline Serum FFAs & 1.414 & $1.237-1.617$ & $<0.001$ & 1.273 & $1.087-1.492$ & 0.003 & 1.939 & $1.388-2.710$ & $<0.001$ \\
\hline PP & 1.028 & $1.009-1.047$ & 0.003 & 1.014 & $0.992-1.036$ & 0.216 & 1.076 & $1.033-1.120$ & $<0.001$ \\
\hline Age & 1.044 & $1.012-1.077$ & 0.007 & 1.033 & $0.997-1.071$ & 0.073 & 1.093 & $1.017-1.174$ & 0.015 \\
\hline eGFR & 1.000 & $0.986-1.014$ & 0.950 & 0.994 & $0.977-1.011$ & 0.469 & 1.011 & $0.984-1.039$ & 0.434 \\
\hline $\mathrm{HbA1c}$ & 1.027 & $0.863-1.222$ & 0.766 & & & & & & \\
\hline
\end{tabular}

$\mathrm{PP}$, pulse pressure; $\mathrm{EGFR}$, estimated glomerular filtration rate

$\mathrm{CI}=1.237-1.617, p<0.001$, Model 1 ), with a $41.4 \%$ increased odds of SCAC for every $1 \mathrm{mmol} / \mathrm{dl}$ increase in serum FFA. Model 2 revealed that in the non-DM group $(\mathrm{n}=302)$, the relationship was weakened $(\mathrm{OR}=1.273$, 95\% CI $=1.087-1.492, p=0.003$, Model 2). As for the DM group $(n=124)$, the association was the strongest ( $\mathrm{OR}=1.939,95 \% \mathrm{CI}=1.388-2.710, p<0.001$, Model 3$)$, with a $93.9 \%$ increased odds of SCAC for every $1 \mathrm{mmol} /$ dl increase in serum FFA after adjusting for traditional cardiovascular risk factors. Serum FFA levels in the DM group were higher compared with the non-DM group (Additional file 1: Table S1).

\section{Predictive ability of FFAs for SCAC}

The predictive ability of serum FFAs for SCAC was assessed by ROC curve analysis (Fig. 4). Similar to the logistic regression results, the AUC was 0.695 (CI 0.641$0.750, p<0.001$, cutoff value: $5.0 \mathrm{mmol} / \mathrm{dl}$; sensitivity: $79.3 \%$; specificity: $51.6 \%$ ) in the total population. In the non-DM group, the AUC was 0.649 (CI 0.580-0.718, $p<0.001$, cutoff value: $5.0 \mathrm{mmol} / \mathrm{dl}$; sensitivity: $71.2 \%$; specificity: $53.0 \%)$. The AUC value (0.775) was the highest in the DM group (CI 0.690-0.859, $p<0.001$, cutoff value: $5.3 \mathrm{mmol} / \mathrm{dl}$; sensitivity: $87.5 \%$; specificity: $53.6 \%$ ).

\section{Discussion}

The main findings of the present study were as follows: (1) Serum FFAs were independently associated with SCAC, and the relationship was enhanced in the DM group. (2) DM patients had a higher risk of elevated FFAs and SCAC. (3) Serum FFA levels may have some predictive capacity for SCAC, especially in the DM subgroup.

The relationship between serum FFA levels and arterial calcification has been discussed in a few studies. Brooder MR et al. [22] found that saturated FFAs can increase medial calcification represented by arterial stiffness in a rat model. The results support our view, although they used palmitate, a component of FFAs abundantly present in serum. In contrast, Conway et al. [26] and Ormseth et al. [10] did not find any relationship between serum FFA levels and CAC. This discrepancy may be due to the inclusion criteria since Conway et al. [26] and Ormseth et al. [10] enrolled either younger T1DM patients or rheumatic patients, while we enrolled all patients with coronary artery disease in the real world. Furthermore, given that saturated FFAs enhance and unsaturated FFAs reduce vascular calcification and arterial stiffness [30, 31], we chose whole FFAs and evaluated the relationship between serum FFA levels and SCAC in our study.

Some previous studies examined the extent of CAC with CT $[10,26]$. In this study, we used IVUS to examine the level of calcification. IVUS is the gold standard for CAC detection, with high sensitivity and specificity $[9,32]$. Compared with CT, IVUS can also provide more details of calcification, such as morphology, position, arc and length [33], which increased the accuracy and reliability of the results of our study.

DM is correlated with CAC extent [2]. Our data showed that $37.7 \%$ of the patients in the SCAC group suffered from T2DM compared with $26.2 \%$ of the patients in the non-SCAC group. From our ROC analysis, serum FFAs showed certain predictive potential of SCAC, with the best in the DM subgroup. In line with this finding, Schauer et al. [11] reported that a high FFA level coupled with insulin resistance can predict the extent of $\mathrm{CAC}$, and may contribute to the increased risk of cardiovascular disease in patients with T1DM. Experimental studies showed that a high level of serum FFAs was associated with insulin resistance and the development of DM [11-13]. Meanwhile, insulin resistance, an important characteristic of DM, plays a crucial role in the process of calcification in DM patients [11, 34]. Hence, we speculated that FFAs may promote the progression of CAC to an advanced stage by affecting the insulin activity in DM patients. This hypothesis needs further investigation.

In this study, in addition to FFAs, pulse pressure (PP) and age also served as predictors of SCAC, which was in line with previous studies $[35,36]$. CKD, a traditionally recognized risk factor for $\mathrm{CAC}$, was not found to be significantly related to SCAC in our study, which may be 


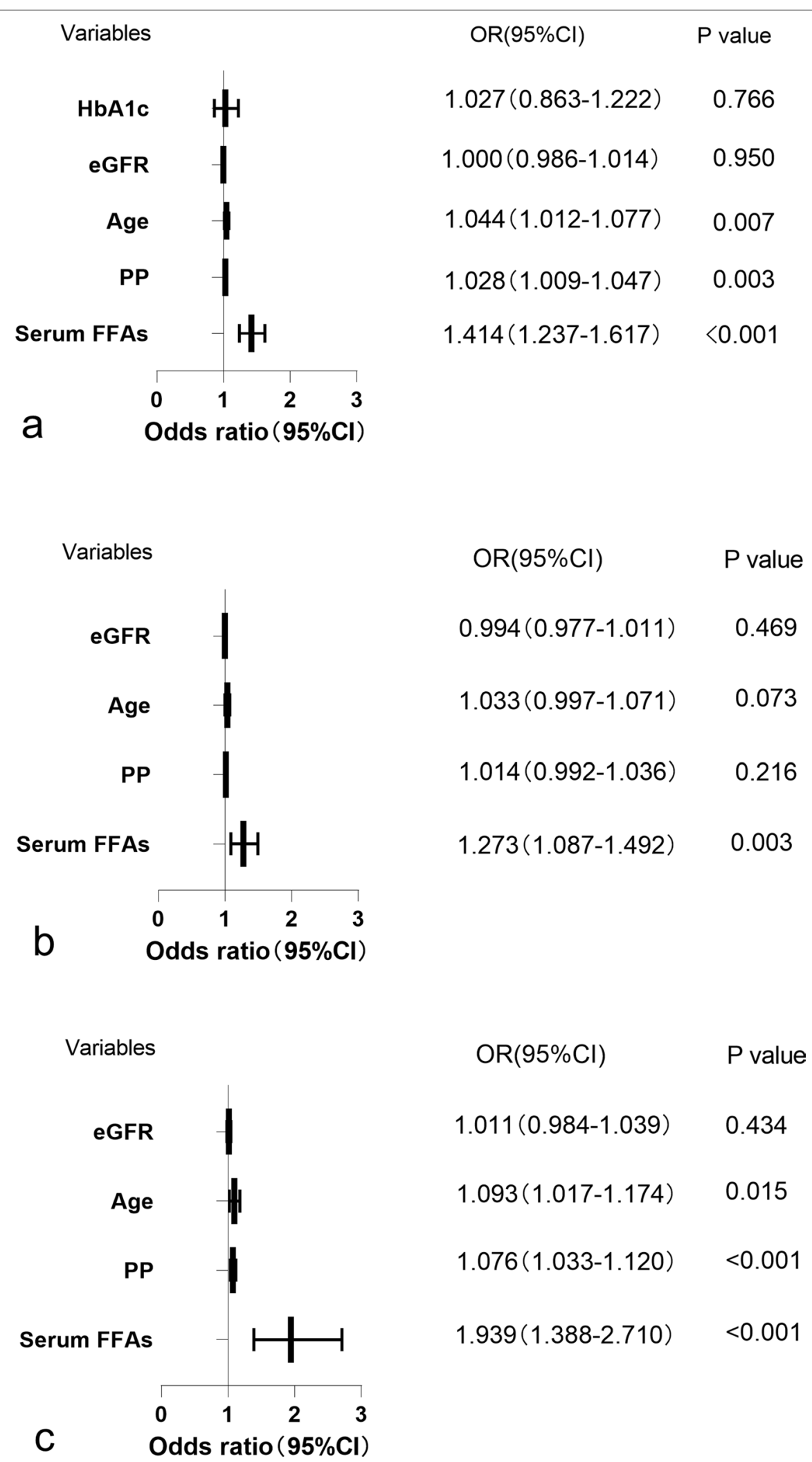

Fig. 3 The Forest plot between SCAC and the risk factors in the whole population (a), non-DM subgroup (b) and DM subgroup (c). Values on the right side are reported as the odds ratio with the respective $95 \%$ confidence interval in parenthesis 

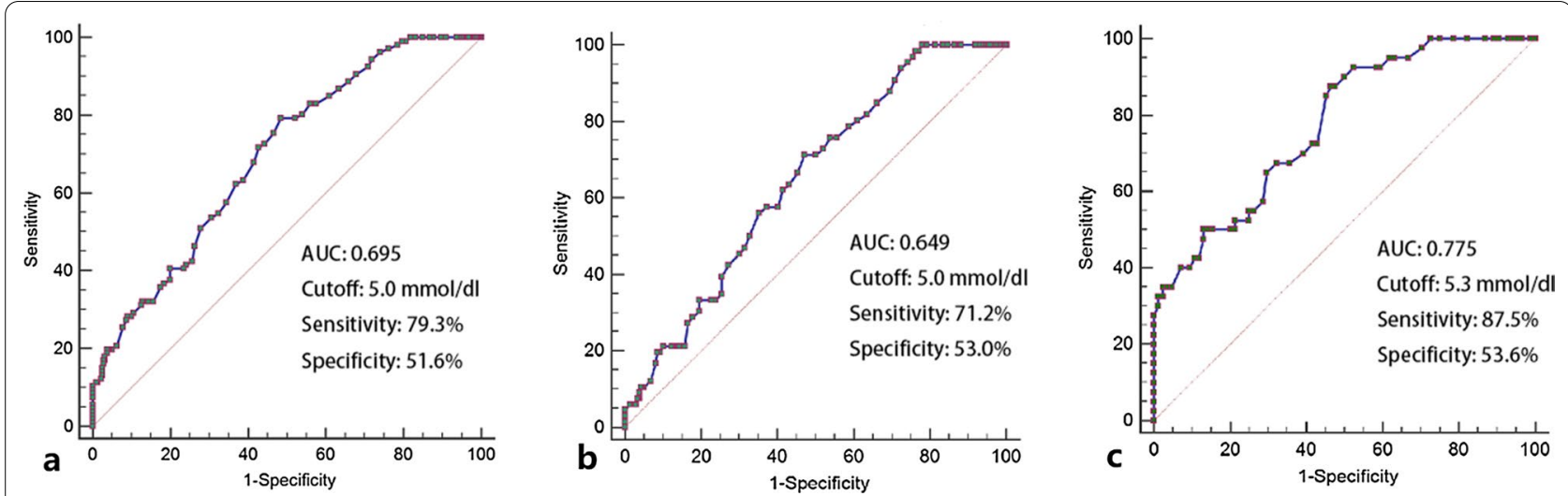

Fig. 4 ROC curve analysis of Serum FFA levels in distinguishing SCAC in the whole population (a), non-DM subgroup (b) and DM subgroup (c)

due to our exclusion of patients with stage 4 to 5 CKD or patients treated with dialysis who suffered greatly from SCAC $[37,38]$.

This study had some limitations. First, this was a retrospective, single-center study with a relatively small sample size, and we focused only on the most severe coronary vessel without evaluation of the other coronaries. Second, we collected only serum fasting FFAs without postprandial FFA levels or various components of serum FFAs.

\section{Conclusions}

Our present study indicated that serum FFAs may have some potential for predicting the severity of CAC, especially in DM patients. More prospective studies with large sample size are needed to further demonstrate the predictive value of FFA in CAC.

\section{Abbreviations \\ FFA: Free fatty acids; DM: Diabetes mellitus; CAC: Coronary artery calcification; IVUS: Intravascular ultrasound; SCAC: Severe coronary artery calcification; ROC: Receiver operating characteristic; AUC: Area under the curves; OR: Odds ratio; $\mathrm{Cl}$ : Confidence interval; CKD: Chronic kidney disease; PCl: Percutaneous coro- nary intervention; CT: Computed tomography; CAG: Coronary angiography; OCT: Optical coherence tomography; CAD: Coronary artery disease; CABG: Coronary artery bypass grafting; NYHA: New York Heart Association; eGFR: Estimated glomerular filtration rate; BMI: Body mass index; HbA1c: Glycated hemoglobin; eGFR: Estimated glomerular filtration rate; CSA: Cross-sectional area; EEM: External elastic membrane; LA: Lumen area; MLA: Minimum lumen area;T1DM: Type 1 diabetes mellitus.}

\section{Supplementary Information}

The online version contains supplementary material available at https://doi. org/10.1186/s12872-021-02152-w.

Additional file 1: Table S1. Baseline clinical characteristics between nondiabetic (Non-DM) and diabetic (DM) groups.
Acknowledgements

We appreciate all the support from participants who took in the design and implementation of the study.

\section{Authors' contributions}

Professor C.Q.W. and Y.Q.F. designed the study; Y.X.X. collected and analyzed the data and wrote the manuscript. J.F.Z. and C.Q.W. reviewed and edited the manuscript. All authors approved the final version of the paper.

\section{Funding}

The study was supported by the Shanghai Science and Technology Commission (18411950500).

\section{Availability of data and material}

The datasets analyzed in the study can be obtained from the corresponding author on reasonable request.

\section{Declarations}

Ethics approval and consent to participate

The present study was approved by the Ethics Committee of the Ninth People's Hospital affiliated to Shanghai Jiao Tong University School of Medicine.

\section{Consent for publication}

Not applicable.

\section{Competing interests}

The authors declare no competing interests.

Received: 18 January 2021 Accepted: 7 July 2021

Published online: 15 July 2021

\section{References}

1. Shanahan CM, Crouthamel MH, Kapustin A, Giachelli CM. Arterial calcification in chronic kidney disease: key roles for calcium and phosphate. Circ Res. 2011;109(6):697-711.

2. Nicoll R, Zhao Y, Wiklund U, Diederichsen A, Mickley H, Ovrehus K, et al. Diabetes and male sex are key risk factor correlates of the extent of coronary artery calcification: a Euro-CCAD study. J Diabetes Complicat. 2017;31(7):1096-102.

3. Goel M, Wong ND, Eisenberg H, Hagar J, Kelly K, Tobis JM. Risk factor correlates of coronary calcium as evaluated by ultrafast computed tomography. Am J Cardiol. 1992;70(11):977-80.

4. Wong ND, Kouwabunpat D, Vo AN, Detrano RC, Eisenberg H, Goel $\mathrm{M}$, et al. Coronary calcium and atherosclerosis by ultrafast computed tomography in asymptomatic men and women: relation to age and risk factors. Am Heart J. 1994;127(2):422-30. 
5. Yamamoto H, Imazu M, Hattori Y, Tadehara F, Yamakido M, Nakanishi T, et al. Predicting angiographic narrowing $>$ or $=50 \%$ in diameter in each of the three major arteries by amounts of calcium detected by electron beam computed tomographic scanning in patients with chest pain. Am J Cardiol. 1998;81 (6):778-80.

6. Guedeney P, Claessen BE, Mehran R, Mintz GS, Liu M, Sorrentino S, et al. Coronary calcification and long-term outcomes according to drugeluting stent generation. JACC Cardiovasc Interv. 2020;13(12):1417-28.

7. Mitchell JD, Paisley R, Moon P, Novak E, Villines TC. Coronary artery calcium and long-term risk of death, myocardial infarction, and stroke: the Walter Reed Cohort Study. JACC Cardiovasc Imaging. 2018;11(12):1799-806.

8. Detrano R, Guerci AD, Carr JJ, Bild DE, Burke G, Folsom AR, et al. Coronary calcium as a predictor of coronary events in four racial or ethnic groups. N Engl J Med. 2008;358(13):1336-45.

9. Wang X, Matsumura M, Mintz GS, Lee T, Zhang W, Cao Y, et al. In vivo calcium detection by comparing optical coherence tomography, intravascular ultrasound, and angiography. JACC Cardiovasc Imaging. 2017;10(8):869-79.

10. Ormseth MJ, Swift LL, Fazio S, Linton MF, Chung CP, Raggi P, et al. Free fatty acids are associated with insulin resistance but not coronary artery atherosclerosis in rheumatoid arthritis. Atherosclerosis. 2011;219(2):869-74

11. Schauer IE, Snell-Bergeon JK, Bergman BC, Maahs DM, Kretowski A, Eckel $\mathrm{RH}$, et al. Insulin resistance, defective insulin-mediated fatty acid suppression, and coronary artery calcification in subjects with and without type 1 diabetes: the CACTI study. Diabetes. 2011;60(1):306-14.

12. Johnston LW, Harris SB, Retnakaran R, Giacca A, Liu Z, Bazinet RP, et al. Association of NEFA composition with insulin sensitivity and beta cell function in the Prospective Metabolism and Islet Cell Evaluation (PROMISE) cohort. Diabetologia. 2018;61(4):821-30.

13. Stefan N, Haring HU. Circulating fetuin-A and free fatty acids interact to predict insulin resistance in humans. Nat Med. 2013;19(4):394-5.

14. Zhang HW, Zhao X, Guo YL, Zhu CG, Wu NQ, Sun J, et al. Free fatty acids and cardiovascular outcome: a Chinese cohort study on stable coronary artery disease. Nutr Metab (Lond). 2017;14:41.

15. Breitling LP, Rothenbacher D, Grandi NC, Marz W, Brenner H. Prognostic usefulness of free fatty acids in patients with stable coronary heart disease. Am J Cardiol. 2011;108(4):508-13.

16. Schrieks IC, Nozza A, Stahli BE, Buse JB, Henry RR, Malmberg K, et al. Adiponectin, free fatty acids, and cardiovascular outcomes in patients with type 2 diabetes and acute coronary syndrome. Diabetes Care. 2018;41(8):1792-800.

17. Kan Y, Wang H, Lu J, Lin Z, Lin J, Gong P. Significance of plasma free fatty acid level for assessing and diagnosing acute myocardial infarction. Biomark Med. 2020;14(9):739-47.

18. Degoricija V, Trbusic M, Potocnjak I, Radulovic B, Pregartner G, Berghold $A$, et al. Serum concentrations of free fatty acids are associated with 3-month mortality in acute heart failure patients. Clin Chem Lab Med. 2019;57(11):1799-804.

19. Djousse L, Benkeser D, Arnold A, Kizer JR, Zieman SJ, Lemaitre RN, et al. Plasma free fatty acids and risk of heart failure: the Cardiovascular Health Study. Circ Heart Fail. 2013;6(5):964-9.

20. Masuda M, Ting TC, Levi M, Saunders SJ, Miyazaki-Anzai S, Miyazaki M. Activating transcription factor 4 regulates stearate-induced vascular calcification. J Lipid Res. 2012;53(8):1543-52.

21. Shiozaki Y, Okamura K, Kohno S, Keenan AL, Williams K, Zhao X, et al. The CDK9-cyclin T1 complex mediates saturated fatty acid-induced vascular calcification by inducing expression of the transcription factor CHOP. J Biol Chem. 2018;293(44):17008-20.

22. Brodeur MR, Bouvet C, Barrette M, Moreau P. Palmitic acid increases medial calcification by inducing oxidative stress. J Vasc Res. 2013;50(5):430-41.

23. Kageyama A, Matsui H, Ohta M, Sambuichi K, Kawano H, Notsu T, et al. Palmitic acid induces osteoblastic differentiation in vascular smooth muscle cells through ACSL3 and NF-kappaB, novel targets of eicosapentaenoic acid. PLOS ONE. 2013;8(6):e68197.

24. Ting TC, Miyazaki-Anzai S, Masuda M, Levi M, Demer LL, Tintut Y, et al. Increased lipogenesis and stearate accelerate vascular calcification in calcifying vascular cells. J Biol Chem. 2011;286(27):23938-49.

25. Masuda M, Miyazaki-Anzai S, Keenan AL, Okamura K, Kendrick J, Chonchol M, et al. Saturated phosphatidic acids mediate saturated fatty acid-induced vascular calcification and lipotoxicity. J Clin Investig. 2015;125(12):4544-58.

26. Conway B, Evans RW, Fried L, Kelsey S, Edmundowicz D, Orchard TJ. Free fatty acids are associated with pulse pressure in women, but not men, with type 1 diabetes mellitus. Metabolism. 2009;58(9):1215-21.

27. Judkins MP. Selective coronary arteriography. I. A percutaneous transfemoral technic. Radiology. 1967;89(5):815-24.

28. Windecker S, Kolh P, Alfonso F, Collet JP, Cremer J, Falk V, et al. 2014 ESC EACTS Guidelines on myocardial revascularization: The Task Force on Myocardial Revascularization of the European Society of Cardiology (ESC) and the European Association for Cardio-Thoracic Surgery (EACTS) developed with the special contribution of the European Association of Percutaneous Cardiovascular Interventions. Eur Heart J. 2014;35(37):2541-619.

29. Mintz GS, Nissen SE, Anderson WD, Bailey SR, Erbel R, Fitzgerald PJ, et al. American college of cardiology clinical expert consensus document on standards for acquisition, measurement and reporting of Intravascular Ultrasound Studies (IVUS). A report of the American College of Cardiology Task Force on Clinical Expert Consensus Documents. J Am Coll Cardiol. 2001;37(5):1478-92.

30. Monahan KD, Feehan RP, Blaha C, McLaughlin DJ. Effect of omega-3 polyunsaturated fatty acid supplementation on central arterial stiffness and arterial wave reflections in young and older healthy adults. Physiol Rep. 2015;3(6):e12438.

31. Heine-Broring RC, Brouwer IA, Proenca RV, van Rooij FJ, Hofman A, Oudkerk M, et al. Intake of fish and marine n-3 fatty acids in relation to coronary calcification: the Rotterdam Study. Am J Clin Nutr. 2010;91(5):1317-23.

32. Friedrich GJ, Moes NY, Muhlberger VA, Gabl C, Mikuz G, Hausmann D, et al. Detection of intralesional calcium by intracoronary ultrasound depends on the histologic pattern. Am Heart J. 1994;128(3):435-41.

33. Okabe T, Mintz GS, Weigold WG, Roswell R, Joshi S, Lee SY, et al. The predictive value of computed tomography calcium scores: a comparison with quantitative volumetric intravascular ultrasound. Cardiovasc Revasc Med. 2009;10(1):30-5.

34. Yamazoe M, Hisamatsu T, Miura K, Kadowaki S, Zaid M, Kadota A, et al Relationship of insulin resistance to prevalence and progression of coronary artery calcification beyond metabolic syndrome components: Shiga Epidemiological Study of subclinical atherosclerosis. Arterioscler Thromb Vasc Biol. 2016:36(8):1703-8.

35. Wu X, Geng YJ, Chen Z, Krishnam MS, Detrano R, Liu H, et al. Pulse pressure correlates with coronary artery calcification and risk for coronary heart disease: a study of elderly individuals in the rural region of Southwest China. Coron Artery Dis. 2019;30(4):297-302.

36. Liu Y, Fu S, Bai Y, Luo L, Ye P. Relationship between age, osteoporosis and coronary artery calcification detected by high-definition computerized tomography in Chinese elderly men. Arch Gerontol Geriatr. 2018;79:8-12.

37. Garland JS, Holden RM, Groome PA, Lam M, Nolan RL, Morton AR, et al. Prevalence and associations of coronary artery calcification in patients with stages 3 to 5 CKD without cardiovascular disease. Am J Kidney Dis. 2008;52(5):849-58.

38. Sigrist M, Bungay P, Taal MW, McIntyre CW. Vascular calcification and cardiovascular function in chronic kidney disease. Nephrol Dial Transplant. 2006;21(3):707-14

\section{Publisher's Note}

Springer Nature remains neutral with regard to jurisdictional claims in published maps and institutional affiliations. 